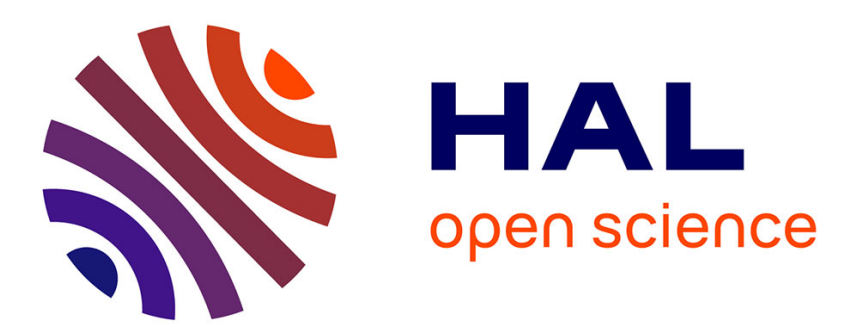

\title{
Appareillage pour l'étude des propriétés rhéologiques des matériaux soumis à des vitesses de déformation rationnelle constante
}

H. Lecoanet, C. Oudet, C. Oytana

\section{- To cite this version:}

H. Lecoanet, C. Oudet, C. Oytana. Appareillage pour l'étude des propriétés rhéologiques des matériaux soumis à des vitesses de déformation rationnelle constante. Revue de Physique Appliquée, 1971, 6 (2), pp.117-119. 10.1051/rphysap:0197100602011700 . jpa-00243510

HAL Id: jpa-00243510

https://hal.science/jpa-00243510

Submitted on 1 Jan 1971

HAL is a multi-disciplinary open access archive for the deposit and dissemination of scientific research documents, whether they are published or not. The documents may come from teaching and research institutions in France or abroad, or from public or private research centers.
L'archive ouverte pluridisciplinaire HAL, est destinée au dépôt et à la diffusion de documents scientifiques de niveau recherche, publiés ou non, émanant des établissements d'enseignement et de recherche français ou étrangers, des laboratoires publics ou privés. 


\title{
APPAREILLAGE POUR L'ÉTUDE DES PROPRIÉTÉS RHÉOLOGIQUES DES MATÉRIAUX SOUMIS A DES VITESSES DE DÉFORMATION RATIONNELLE CONSTANTE
}

\author{
H. LECOANET, C. OUDET et C. OYTANA
}

Laboratoire de Mécanique Appliquée, Associé au C. N. R. S., Faculté des Sciences de Besançon, La Bouloie, 25, Besançon

(Reçu le 16 novembre 1970)

\begin{abstract}
Résumé. - Description d'un appareillage permettant d'étudier les propriétés rhéologiques des matériaux en traction et compression avec vitesse de déformation rationnelle constante. Un moteur asservi en vitesse est piloté par un générateur d'exponentielles à valeurs initiales réglables. Il entraîne le selsyn de référence d'une machine de traction-compression classique.

Abstract - We describe an apparatus allowing the study of the traction or compression rheological properties of materials with a constant true strain rate. A variable speed controlled motor is drived by an exponential functions generator with adjustable initial values. It drives the reference selsyn of a classical traction compression machine.
\end{abstract}

I. Intérêt de l'appareillage. - De nombreuses études concernant la superplasticité sont actuellement en cours dans divers laboratoires [1]. Les matériaux superplastiques sont caractérisés par une grande ductilité et une forte dépendance de la contrainte d'écoulement vis-à-vis de la vitesse de déformation. Dans ces cas on introduit la déformation logarithmique ou déformation rationnelle [2]. Au lieu de rapporter l'allongement d'un échantillon à sa longueur initiale $l_{0}$ on le rapporte à la dimension actuelle et la déformation correspondant au passage de la longueur $l_{0}+\Delta l$ est :

$$
\bar{\varepsilon}=\int_{l_{0}}^{l_{0}}+\Delta l \frac{\mathrm{d} l}{l}=\log \frac{l_{0}+\Delta l}{l_{0}}=\log (1+\varepsilon) .
$$

Pour l'étude en traction ou compression de matériau présentant à la fois une grande ductilité et un fort caractère visqueux l'utilisation d'une vitesse de traverse mobile constante s'avère insuffisante. En effet, soit une éprouvette de longueur instantanée $l$ et de section instantanée $A$ dont le comportement rhéologique est régit par l'équation :

$$
\sigma=K \bar{\varepsilon}^{\prime m}
$$

(c'est le cas des alliages superplastiques avec $0,3<m<0,8)$. Si on teste cet échantillon avec une vitesse d'allongement ou de compression $v$ on a :

$$
\begin{aligned}
& \varepsilon^{\prime}=\frac{v}{l_{0}+x} \\
& \sigma=\frac{K v^{m}}{\left(l_{0}+x\right)^{m}} \\
& F=\frac{K v^{m} A_{0} l_{0}}{\left(l_{0}+x\right)^{m+1}} .
\end{aligned}
$$

L'indice 0 correspond aux valeurs initiales de l'éprouvette, $x$ étant le déplacement de la traverse, et $F$ la force nécessaire au maintien de $v$, force qui est indiquée par la cellule de mesure de charges, $F$ et $\sigma$ dépendent donc non seulement de $v$ et $m$ mais également de la longueur initiale de l'éprouvette et de $x$ (compte non tenu de l'écrouissage) et ce par une relation trop complexe pour permettre de remonter facilement à l'équation (1).

II. Principe de l'appareillage. - 1. OBJECTIF A ATTEINDRE. - On veut

$$
\varepsilon^{\prime}=\frac{1}{l} \frac{\mathrm{d} l}{\mathrm{~d} t}=C
$$

où $C$ est la vitesse de déformation rationnelle choisie $(C>0$ pour un essai de traction et $C<0$ pour une compression). En fait on va asservir la vitesse de déplacement $v$ de l'extrémité de l'éprouvette (et non la vitesse de déformation) qui est celle de la traverse mobile. Il est cependant possible avec la technique utilisée d'asservir le $\bar{\varepsilon}$ ' de la partie utile de l'éprouvette en utilisant un extensomètre.

En intégrant (4) on doit avoir :

$$
v=v_{0} \mathrm{e}^{C t}
$$

(étant donné que $v=\mathrm{d} l / \mathrm{d} t) v_{0}$ étant déterminé par la condition initiale

$$
\bar{\varepsilon}^{\prime}=\frac{l}{l_{0}} v_{0}=C .
$$

Il faut donc pouvoir :

a) afficher une valeur de $C$,

b) pour une vitesse de déformation rationnelle $C$ donnée, ajuster la vitesse $v_{0}$ de la traverse mobile selon la longueur initiale de l'éprouvette à tester. 
2. Principe DE L'APPAReIllage. - L'appareillage est conçu pour être adapté sur une machine électromécanique type Instron. Dans celle-ci un Selsyn de référence commande la position de la traverse mobile et peut être piloté (entre autres) de l'extérieur. On a alors $x=K \theta$ où $\theta$ est la position angulaire du selsyn. Pour réaliser les conditions (7) et (8) la vitesse de rotation du selsyn doit alors être

$$
\omega=\frac{l_{0}}{k} C \mathrm{e}^{c t} \text {. }
$$

Pour ce faire, le selsyn est entraîné par un moteur asservi en vitesse qui permet de commander la vitesse de rotation de l'ensemble par une tension $V$ (nous avons utilisé le système "Motomatic » de la Société Electrocraft dont le «maître-contrôle » a été adapté à la commande par tension extérieure).

La tension $V$ est fournie par un générateur d'exponentielle $V=V_{0} \mathrm{e}^{C t}(C>0$ ou $<0)$ permettant d'afficher indépendamment $V_{0}$ et $C$. Si pour le servomoteur $\omega=\alpha V$, la valeur de $C$ sélectionnée donnera la vitesse de déformation rationnelle et pour une éprouvette de longueur initiale $l_{0}$ il faudra imposer

$$
V_{0}=\frac{l_{0}}{\alpha k} C
$$

pour respecter la condition initiale (8).

III. Réalisation. - On dispose sur un portique de machine Instron de 3 valeurs possibles de $k$;

$$
k=\frac{1}{3,6 \pi 10^{\beta}} \text { avec } \beta=0 ; 1 ; 2,
$$

qui jointes aux valeurs de $\omega$ compatibles avec le selsyn de la machine permettent de déterminer les valeurs de $\alpha$ à utiliser (choix du réducteur du moteur générateur). Les valeurs de la vitesse de déformation rationnelle intéressantes pour la plupart des matériaux sont telles que

$$
10^{-5} \mathrm{~s}^{-1}<C<10^{-1} \mathrm{~s}^{-1}
$$

et déterminent le choix des constantes de temps des tensions exponentielles à produire. On trouve figure 1 le montage utilisé pour fabriquer les exponentielles :

$C<0$ s'obtient par l'intermédiaire d'un circuit $R C$. La tension utilisée est prise aux bornes de la résistance pendant la charge de la capacité sous tension constante. Cinq valeurs de $C$ et deux valeurs de $R$ permettent d'obtenir 10 vitesses de déformations rationnelles. La valeur $V_{0}$ est la tension appliquée aux bornes du circuit $R C$ et se règle au moyen d'un potentiomètre 10 tours, $100 \mathrm{k} \Omega: \mathrm{P}_{1}$. Il est possible d'obtenir une variation continue de la constante de temps en remplaçant les 2 résistances ( $60 \mathrm{M} \Omega$ et $120 \mathrm{M} \Omega$ ) du circuit $R C$ par des potentiomètres.

Pour réaliser $C>0$ on utilise un générateur de fonction non linéaire mettant en œuvre un transistor dans le circuit d'entrée d'un amplificateur opérationnel, suivant une disposition bien connue. En effet, pour certains semi-conducteurs le courant est une fonction exponentielle de la tension appliquée à la fonction. L'amplificateur opérationnel recopie ce courant dans la résistance $R$ de contre-réaction, d'où à la sortie une tension exponentielle, d'un niveau réglable à volonté par $R$. La tension d'entrée, appliquée à la jonction est une rampe obtenue en intégrant une tension constante.

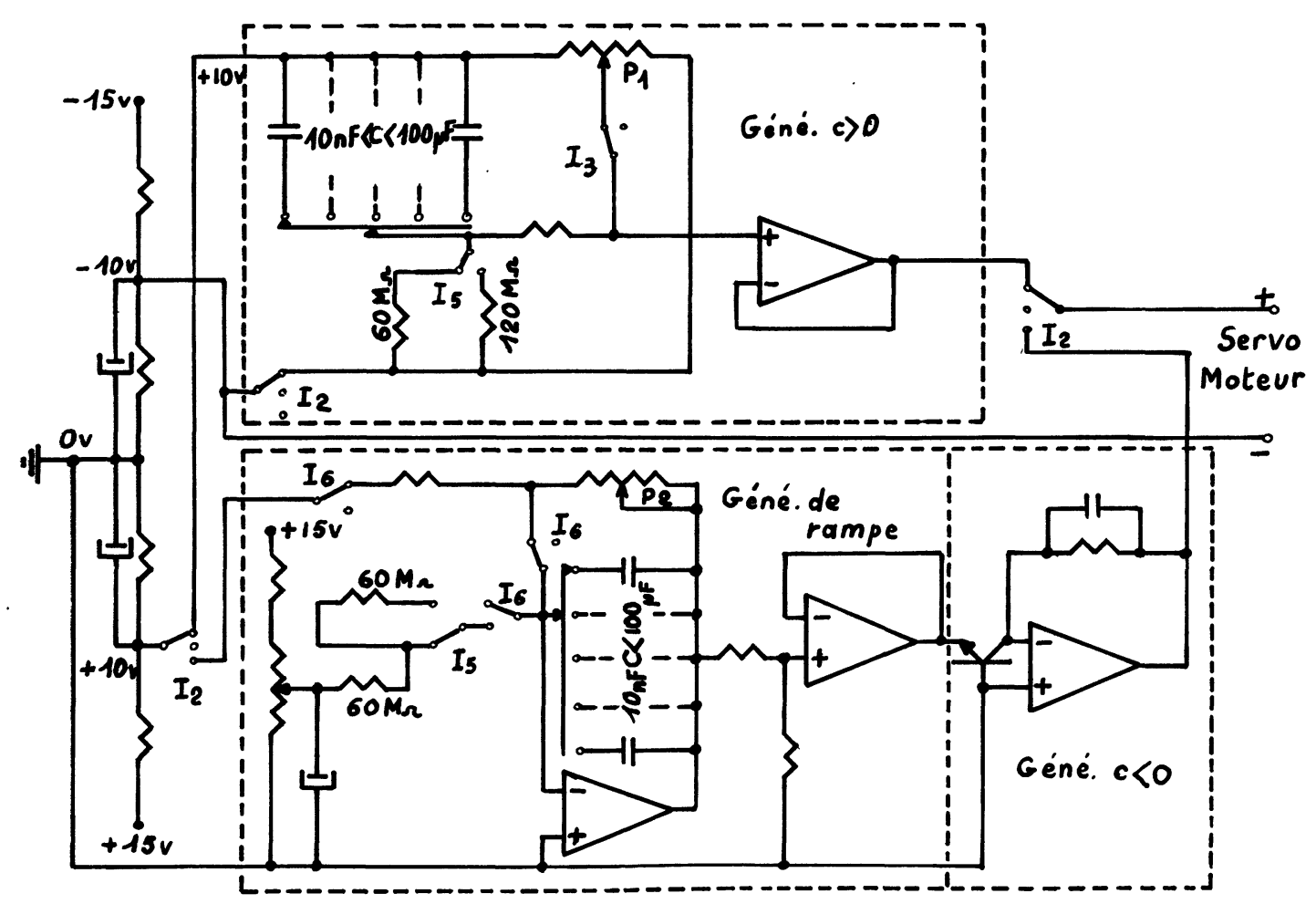

FIG. 1. 
Les différentes valeurs de $C$ correspondent aux différentes valeurs de la constante d'intégration (10 sont disponibles et, comme pour $C<0$, il est possible de disposer d'une évolution continue), le réglage de $V_{0}$ s'obtient en ajustant la tension à intégrer à l'aide d'un potentiomètre 10 tours : $P_{2}$. Notons qu'en série avec la sortie du générateur de fonctions exponentielles et la commande du servomoteur on dispose une source de tension continue réglable entre 0 et $1 \mathrm{~V}$ (non représentée sur le schéma) pour compenser le décalage d'origine de la caractéristique $\omega=f(V)$ de l'ensemble « Motomatic » utilisé.

IV. Résultats. - La vérification et l'étalonnage de cette chaîne cinématique sont faits par enregistrement du déplacement de la traverse en fonction du temps par l'intermédiaire d'un capteur de déplacement à grande course.

Ils montrent que la vitesse de traverse obéit à la relation (7) à $5 \%$ près (pour les dimensions d'éprouvettes utilisables). Dans ces cas les allongements rela- tifs permis par l'appareillage sont de l'ordre de 10 pour toutes les valeurs de $C$, ce qui est largement suffisant pour la plupart des expériences [4]. Ils sont en fait surtout limités par la machine de traction elle-même. On a en effet la relation :

$$
\frac{l-l_{0}}{l_{0}}=\frac{v}{v_{0}}=\mathrm{e}^{c t}
$$

qui montre que la vitesse $v$ qu'il faut atteindre pour observer un allongement relatif donné est proportionnel à $V_{0}=l_{0} C$. Pour les grandes valeurs de $C, v$ peut alors dépasser la capacité en puissance ou vitesse de la machine, ce qui limite la dynamique de l'installation.

Notons enfin, qu'au-dessous de $C<10^{-5} \mathrm{~s}^{-1}$ l'utilisation des vitesses de traverses constantes s'avère généralement suffisante.

Nous tenons à remercier $M$. Cosme, Technicien du C. N. R. S., pour l'aide qu'il nous a apportée dans la réalisation et la mise au point de l'appareillage électronique.

\section{Bibliographie}

[1] Oelschagel, Superplasticity in metals, Bull. Japan Inst. Metals, 1957, VI, 11-21.

[2] Ludwick (P.), Elemente der technologischen Mechanik, Berlin, 1909.

[3] Lautenschlager (E.), Brittain (J. O.), Constant true strain rate apparatus, Revue of scientific instruments, 1968, 39, 1563-1565.

[4] Honeycombe (R. W. R.), The plastic deformation metals Edward Arnold, 1968; ThOMSEN (E. G.), YANG (C. T.), Kobayashi (S.), Plastic deformation in metal progressing Macmillan, 1965. 